\title{
A COMPLETE ELEMENTARY PROOF THAT HILBERT SPACE IS HOMEOMORPHIC TO THE COUNTABLE INFINITE PRODUCT OF LINES ${ }^{1}$
}

\author{
BY R. D. ANDERSON AND R. H. BING
}

1. Introduction. In this paper we give a complete and self-contained proof that real Hilbert space, $l_{2}$, is homeomorphic to the countable infinite product of lines, $s$; symbolically $l_{2} \sim s$. We assume only that the reader understands the material of a first year course in topology: for example, elementary notions of complete metric spaces, product spaces, continuity, homeomorphisms, and the Tietze Extension Theorem. While the treatment is elementary, the arguments are not necessarily simple. The reader should be prepared to draw figures and verify continuity statements.

Among those who read an earlier version of this paper we particularly thank Frederic Ancel, William Barit, William Jaco, Dallas Webster, and James West who made very useful comments.

We define $l_{2}$ as the space of square summable sequences of realsthat is, $l_{2}=\left\{\left(x_{i}\right)_{i>0} \mid x_{i}\right.$ is a real number and $\left.\sum x_{i}^{2}<\infty\right\}$. For $x, y \in l_{2}$, the distance between $x=\left(x_{i}\right)_{i>0}$ and $y=\left(y_{i}\right)_{i>0}$ is defined by $d(x, y)$ $=\left[\sum\left(x_{i}-y_{i}\right)^{2}\right]^{1 / 2}$. It is easy to verify that under such a distance function, $l_{2}$ is a complete metric space.

We define $s$ as the space of all sequences $\left(x_{i}\right)_{i>0}$ of reals with the usual product topology. Thus $s=\prod_{i>0} R_{i}$, where for each $i, R_{i}$ is the space of reals. A basis for the topology of $s$ is the collection of sets of the form $\prod_{i>0} U_{i}$, where $U_{i}$ is an open subset of $R_{i}$ and for all but a finite number of the $i$ 's, $U_{i}=R_{i}$.

Since for each positive integer $i$, the real line $R_{i}$ is homeomorphic with the open interval $(-1,1)=I_{i}^{0}$, we may alternatively write $s=\prod_{i>0} I_{i}^{0}$. We find this formulation convenient in $\S 3$.

At times $(\$ \S 7$ and 9$)$ we need to regard $s$ as a complete metric space. A convenient complete metric to use is $d(x, y)=\sum\left(\min \left[1 / 2^{i},\left|x_{i}-y_{i}\right|\right]\right)$. An alternate complete metric is the traditional Fréchet metric $d(x, y)=\sum\left|x_{i}-y_{i}\right| /\left[2^{i}\left(1+\left|x_{i}-y_{i}\right|\right)\right]$. It is immaterial, for our purposes, which we use, so let us suppose for simplicity that we use the first.

1 The preparation of this expository article was supported by the National Science Foundation under grant GN 530 to the American Mathematical Society. The research leading to the paper was partially supported by NSF grant GP-6867 and GP-3857. Submitted by invitation of the editors; received by the editors April 19, 1968. 
The question as to whether $l_{2}$ is homeomorphic to $s$ was raised by Fréchet [10] in his book, Les éspaces abstraits (1928), and also by Banach [5] in his book, Thêorie des opérations linéaires (1932). In an added footnote in [5], Banach erroneously attributed to Mazur a negative answer to the question. More recently, Kadec, Bessaga, Pelczynski, and others have studied homeomorphisms between various infinite dimensional Banach and Fréchet spaces. The work up to about 1964 is summarized by Bessaga in [6]. In the direction of proving that $l_{2} \sim s$, Bessaga and Pelczynski [9] had proved that $l_{2} \sim\left(l_{2} \times s\right)$ and that $l_{2}$ was homeomorphic to the countable product of copies of itself. Kadec [11], [12] proved that all separable infinite dimensional Banach spaces are homeomorphic. In 1966, Anderson [1] proved that $l_{2} \sim s$. This was done by using results in [2] together with the result that $l_{2} \sim\left(l_{2} \times s\right)$. Based on an earlier Bessaga and Pelczynski result cited in [6], the results of Kadec [11] and Anderson [1] established that all separable infinite dimensional Fréchet spaces are homeomorphic to each other.

In the present paper, the result that $l_{2} \sim s$ appears in $\S 3$ as Theorem 1.1, but its proof makes use of lemmas and theorems from later sections. The procedure is summarized in $\$ 10 . \$ 11$ lists open questions.

2. Further definitions and notation. We let $X \backslash Y$ denote the set of all elements of $X$ not in $Y$.

Let $Z$ denote the set of positive integers. For $\alpha \subset Z$, we let $\alpha^{\prime}=Z \backslash \alpha$. We let $s^{\alpha}=\prod_{i \in \alpha} R_{i}$ and $l_{2}^{\alpha}$ denote the space of sequences $\left(x_{i}\right)_{i \in \alpha}$ of reals for which $\sum_{i \in \alpha} x_{i}^{2}<\infty$ with $d(x, y)=\left(\sum_{i \in \alpha}\left(x_{i}-y_{i}\right)^{2}\right)^{1 / 2}$.

The following two propositions are well known and easy to prove.

LEMMA 2.1. If $\alpha$ is a nonempty subset of $Z$ for which $\alpha^{\prime}$ is nonempty, then $s \sim s^{\alpha} \times s^{\alpha^{\prime}}$. If $\alpha$ is infinite, then $s_{2}^{\alpha} \sim s$.

LeMma 2.2. If $\alpha$ is a nonempty subset of $Z$ for which $\alpha^{\prime}$ is nonempty, then $l_{2} \sim l_{2}^{\alpha} \times l_{2}^{\alpha^{\prime}}$. If $\alpha$ is infinite, then $l_{2} \sim l_{2}^{\alpha}$.

We use the convention that if $\alpha$ is an infinite subset of $Z$, then propositions concerning $l_{2}$ and $s$ are considered as applicable to $l_{2}^{\alpha}$ and $s^{\alpha}$.

If $\alpha \subset Z$, let $\tau_{\alpha}$ denote the projection of $l_{2}$ or $s$ onto $l_{2}^{\alpha}$ or $s^{\alpha}$. If $\alpha=\{i\}$, we may write $\tau_{\alpha}$ as $\tau_{i}$.

A set $K$ in $l_{2}$ or $s$ is deficient in the ith direction if $\tau_{i}(K)$ consists of a single element. A set $K$ in $l_{2}$ or $s$ is infinitely deficient if for some infinite set $\alpha \subset Z, \tau_{i}(K)$ consists of a single element for each $i \in \alpha$. In this case we also say that $K$ is deficient with respect to $\alpha$.

We use the term map to denote a continuous function. For $f$ a map of $X$ into $Y$ and $K \subset X$, the notation $f \mid K$ denotes the map $f$ 
restricted to the domain $K$. We use Id to denote the identity homeomorphism.

Let $G$ be an open covering of a space $X, Y$ be a subset of $X$, and $f$ be a map of $Y$ into $X$. We say that $f$ is limited by $G$ if for each $p \in Y$, there exists a $g_{p} \in G$ such that $p \in g_{p}$ and $f(p) \in g_{p}$. The covering $G$ provides a measure as to how close $f$ is to the identity. If $X$ is a metric space, the mesh of $G$ is the least upper bound of the diameters of the elements of $G$.

Suppose $f_{1}, f_{2}, \cdots$ is a sequence of maps such that the limit of $f_{1}, f_{2} \circ f_{1}, \cdots$ exists. We denote this limit by $\lim \left(f_{i} \circ \cdots \circ f_{2} \circ f_{1}\right)_{i>0}$ $=L \prod_{i>0} f_{i}$ and call it the infinite left product of the $f_{i}$.

In $\$ 5$ we define invertible isotopies and adopt notation to be used in working with isotopies. In $\$ 7$ we define an invertibly continuous family of invertible isotopies.

We use $S_{1}$ to denote the set of all points of $l_{2}$ whose distance from the origin is 1 -that is, $S_{1}=\left\{x \in l_{2} \mid \sum x_{i}^{2}=1\right\}$.

We use $E^{i}$ to denote $\left\{x \in l_{2} \mid x_{j}=0\right.$ if $\left.j>i\right\}$. Then $U E^{i}$ is the union of all finite dimensional coordinate planes of $l_{2}$. Sometimes $U E^{i}$ is called infinite dimensional Euclidean space. We note that $l_{2} \backslash U E^{i}=\left\{x \in l_{2} \mid x_{i}\right.$ $\neq 0$ for infinitely many $i\}$.

3. A homeomorphism of $l_{2}$ onto $s$. Throughout this section we regard $s=\prod_{i>0} I_{i}^{0}$ and let $C_{i}=\left\{y \in s \mid \sum y_{j}^{2} \leqq i\right.$ and for each $j>0$, $\left.\left|y_{j}\right| \leqq 1-1 / i\right\}$. Note that $C_{i}$ is compact since it is a closed subset of the product of closed intervals.

The main result of the paper is the following.

THEOREM 3.1. $l_{2} \sim s$.

The plan for showing that $l_{2} \sim s$ is to exhibit homeomorphisms through middle spaces as follows:

$$
l_{2} \backsim l_{2} \backslash \cup E^{i} \backsim S_{1} \cap\left(l_{2} \backslash U E^{i}\right) \backsim s \backslash U C_{i} \backsim s .
$$

The four homeomorphisms are guaranteed by Corollary 9.3, Lemma 3.3, Lemma 3.2, and Corollary 9.4 respectively.

The topology of the first three spaces above is determined by the topology of $l_{2}$ while the topology of the last two is determined by the topology of $s$, so we may start by considering the bridge between $l_{2}$ and $s$, namely the homeomorphism of $S_{1} \cap\left(l_{2} \backslash \cup E^{i}\right)$ onto $s \backslash \cup C_{i}$. This bridge homeomorphism suggests why we chose to define a homeomorphism on $S_{1} \cap\left(l_{2} \backslash \cup E^{i}\right)$ instead of on $l_{2}$.

Lemma 3.2. $S_{1} \cap\left(l_{2} \backslash \cup E^{i}\right) \sim s \backslash \cup C_{i}$. 
Proof. Throughout this proof we denote $S_{1} \cap\left(l_{2} \backslash \cup E^{i}\right)$ by $S_{1}{ }^{*}$. Hence the coordinates of points of both $S_{1}^{*}$ and $s$ lie in $(-1,1)$.

There are many 1-1 functions taking $S_{1}^{*}$ onto a dense subset of $s$. Since most of the coordinates of each point of $S_{1}^{*}$ are close to 0 , one might hope to divide each of these coordinates by a suitable divisor and obtain the corresponding coordinate of the corresponding point of $s$. Let us consider such an elementary function $h: S_{1}{ }^{*} \rightarrow s$ where we let

$$
x=\left(x_{1}, x_{2}, \cdots\right) \in S_{1}^{*}, \text { and } h(x)=\left(y_{1}, y_{2}, \cdots\right) \in s .
$$

We note that $x_{1} \in(-1,1)$ and $y_{1} \in(-1,1)$ so we set $y_{1}=x_{1}$.

Once $x_{1}$ is fixed, $x_{2}$ has the limited domain $\left(-\left(1-x_{1}^{2}\right)^{1 / 2},\left(1-x_{1}^{2}\right)^{1 / 2}\right)$ while $y_{2}$ can still be anywhere in $(-1,1)$. We take the domain of $x_{2}$ linearly on to the domain of $y_{2}$ and thus define

$$
y_{2}=x_{2} /\left(1-x_{1}^{2}\right)^{1 / 2} \text {. }
$$

Continuing in this fashion, we define

$$
y_{i}=x_{i} /\left(1-\sum_{1}^{i-1} x_{j}^{2}\right)^{1 / 2} \text {. }
$$

One may note that the function $h$ we have defined is $1-1$ and coordinate wise continuous. Since $s$ is a product space, coordinatewise continuity into $s$ implies continuity. Hence $h$ is continuous and thus is a map.

One reason for working with $S_{1}^{*}$ rather than $S_{1}$ is that we would have had difficulties trying to avoid dividing by 0 .

In order to study $h^{-1}$, we may solve for $\left(x_{1}, x_{2}, \ldots\right)$ in terms of $\left(y_{1}, y_{2}, \cdots\right)$. One finds that $h^{-1}=g \mid h\left(S_{1}^{*}\right)$ where $g: s \rightarrow l_{2}$ is defined by

$$
x_{i}=y_{i}\left(1-y_{1}^{2}\right)^{1 / 2}\left(1-y_{2}^{2}\right)^{1 / 2} \cdots\left(1-y_{i-1}^{2}\right)^{1 / 2} .
$$

Also, $g$ is $1-1$ and coordinatewise continuous. Coordinatewise convergence in $l_{2}$ does not imply convergence as can be seen by considering the sequence of points such that the $i$ th member of the sequence has all coordinates 0 except the $i$ th which is $1 / 2$. However, it is well known and easy to prove that coordinatewise continuity into $S_{1}$ does imply continuity. Therefore $h^{-1}=g \mid h\left(S_{1}^{*}\right)$ is continuous and $h$ is a homeomorphism. (Incidentally, it may be shown that $g$ defined on all of $s$ is not a homeomorphism.) Another reason for defining the bridge homeomorphism on $S_{1}^{*}$ instead of on $l_{2}$ is that functions into $S_{1}$ are continuous if they are coordinatewise continuous. 
The remaining part of the proof of Lemma 3.2 is to show that $s \backslash h\left(S_{1}^{*}\right)=\cup C_{i}$. This equality is no accident since the definition of $C_{i}$ was chosen only after the definition of $h$.

One finds from inductive use of the equation describing the $x_{i}$ 's in terms of the $y_{i}$ 's that

$$
\sum_{1}^{i} x_{j}^{2}=1-\left(1-y_{1}^{2}\right)\left(1-y_{2}^{2}\right) \cdots\left(1-y_{i}^{2}\right) .
$$

Hence, if $y \in s$, the sum of the squares of the first $i$ coordinates of $g(y)$ is less than 1 . In fact, one finds that

$$
g(s)=\left\{x \in l_{2} \mid \text { for each } i, \sum_{1}^{i} x_{j}^{2}<1\right\}
$$

since for each $x \in l_{2}$ such that for each $i, \sum_{1}^{i} x_{j}^{2}<1$, one can inductively define $y_{i}$ coordinates so that $g(y)=x$. In particular, $S_{1}{ }^{*} \subset g(s)$ and

$$
s \backslash h\left(S_{1}^{*}\right)=g^{-1}\left\{x \in l_{2} \mid \sum_{1}^{\infty} x_{i}^{2}<1\right\} .
$$

To prove that $h\left(S_{1}^{*}\right)=s \backslash \cup C_{i}$, we need only show that if $y \in h\left(S_{1}^{*}\right)$, $\sum_{1}^{\infty} y_{i}^{2}=\infty$ and if $y \in s \backslash h\left(S_{1}^{*}\right), \sum_{1}^{\infty} y_{i}^{2}$ is finite.

If $y \in s \backslash h\left(S_{1}^{*}\right)$ and $g(y)=x$, then $\sum_{1}^{\infty} x_{i}^{2}<1$. Since

$$
\begin{gathered}
y_{i}=x_{i} /\left(1-\sum_{1}^{i-1} x_{j}^{2}\right)^{1 / 2}, \text { then } y_{i}^{2} \leqq x_{i}^{2} /\left(1-\sum_{1}^{\infty} x_{j}^{2}\right) \\
\text { and } \sum y_{i}^{2} \leqq \sum x_{i}^{2} /\left(1-\sum_{1}^{\infty} x_{j}^{2}\right) .
\end{gathered}
$$

Hence, $\sum_{1}^{\infty} y_{i}^{2}$ is finite.

If $y \in h\left(S_{1}^{*}\right)$, we use a plan suggested by Robert Connelly and show that $\sum_{1}^{\infty} y_{i}^{2}=\infty$ by demonstrating that for each integer $n$, $\sum_{n}^{\infty} y_{i}^{2}>1$. This is because

$$
\sum_{n}^{\infty} y_{i}^{2}=\frac{x_{n}^{2}}{\sum_{n}^{\infty} x_{j}^{2}}+\frac{x_{n+1}^{2}}{\sum_{n+1}^{\infty} x_{j}^{2}}+\cdots>\frac{x_{n}^{2}+x_{n+1}^{2}+\cdots}{\sum_{n}^{\infty} x_{j}^{2}}=1 .
$$

LEMMA 3.3. $l_{2} \backslash \cup E^{i} \sim S_{1} \cap\left(l_{2} \backslash \cup E^{i}\right)$.

PRoof. The required homeomorphism is $h_{1} \circ h_{2}$ where $h_{2}$ is the homeomorphism which takes each point $\left(x_{1}, x_{2}, \cdots\right)$ to $\left(1, x_{1}, x_{2}, \cdots\right)$ and for each $x \in h_{2}\left(l_{2}\right), h_{1}(x)$ is the point of $S_{1}$ between $x$ and $(-1,0,0, \cdots)$. 
4. Convergence of homeomorphisms. It is trivial to verify that the product (or composition) of any two homeomorphisms of a space $X$ onto itself is a homeomorphism of $X$ onto itself. Therefore, any finite product of such homeomorphisms is a homeomorphism of $X$ onto itself. However, infinite left products, that is products of the form $\cdots \circ h_{i} \circ \cdots \circ h_{2} \circ h_{1}$, may or may not converge so as to define a homeomorphism of $X$ onto itself or even of a subset of $X$ onto $X$.

Equivalently, we may consider the possible convergence of the sequence $h_{1}, h_{2} \circ h_{1}, \cdots$ of homeomorphisms of $X$ onto itself. We may denote this sequence by $\left(h_{i} \circ \cdots \circ h_{2} \circ h_{1}\right)_{i>0}$. Frequently $h_{i}$ is close to the identity so that members of the sequence are products of the previous element of the sequence by a homeomorphism near the identity.

There are two basic types of procedures which we shall use to establish that sequences of the form $\left(g_{i}\right)_{i>0}=\left(h_{i} \circ \cdots \circ h_{2} \circ h_{1}\right)_{i>0}$ converge to desired homeomorphisms of some subset $Y$ of $X$ onto $X$.

Convergence Procedure I. A simple form of this procedure is represented by the following trivial but useful theorem.

THEOREM 4.1. The sequence $\left(g_{i}\right)_{i>0}$ of homeomorphisms of $X$ onto $X$ converges to a homeomorphism of the subset $Y$ of $X$ onto $X$ provided that

(1) for each $p \in Y$ there exists a neighborhood $U$ of $p$ in $X$ and an integer $n(U)$ such that for each $n>n(U), g_{n}\left|U=g_{n(U)}\right| U$ and

(2) for each $q \in X$ there exists a neighborhood $V$ of $q$ and an integer $n(V)$ such that for each $n>n(V), g_{n}^{-1}\left|V=g_{n(V)}^{-1}\right| V$ and $g_{n}^{-1}(V) \subset Y$.

Observe that if for each $i>0, g_{i}=h_{i} \circ \cdots \circ h_{2} \circ h_{1}$, then the condition that $g_{n}\left|U=g_{n(U)}\right| U$ becomes the condition that $h_{n}$ restricted to $h_{n(U)} \circ \cdots \circ h_{2} \circ h_{1}(U)$ is the identity restricted to $h_{n(U)} \circ \cdots \circ h_{2}$ $\circ h_{1}(U)$. Similarly, the condition $g_{n}^{-1}\left|V=g_{n(V)}^{-1}\right| V$ becomes $h_{n}^{-1} \mid V$ $=\mathrm{Id} \mid V$.

Convergence Procedure II. In some instances it does not seem feasible to construct a desired homeomorphism by a sequential process using Convergence Procedure I. To handle such cases we introduce another procedure. While a simpler version of this convergence procedure has been applied to compact spaces, as far as the authors know, the formulation we give for complete metric spaces is new.

If $G$ is an open covering of a space $X$ and $h$ is a homeomorphism of $X$, we use $h(G)$ to denote the collection of images of elements of $G$. Suppose $\left(h_{i}\right)_{i>0}$ is a sequence of homeomorphisms of a complete metric space $X$ onto itself. We say that $\left(h_{i}\right)_{i>0}$ satisfies the inductive convergence criterion if there is a sequence $\left(G_{i}\right)_{i>0}$ of open coverings of $X$ such that for each positive integer $i$, 
mesh $G_{i}<1 / 2^{i}$,

$\operatorname{mesh}\left(h_{i} \circ \cdots \circ h_{2} \circ h_{1}\right)^{-1}\left(G_{i}\right)<1 / 2^{i}$, and $h_{i+1}$ is limited by $G_{i}$.

THEOREM 4.2. If $\left(h_{i}\right)_{i>0}$ is a sequence of homeomorphisms of a complete metric space $X$ onto itself which satisfies the inductive convergence criterion, then $\left(g_{i}\right)_{i>0}=\left(h_{i} \circ \cdots \circ h_{2} \circ h_{1}\right)_{i>0}$ converges to a homeomorphism of $X$ onto itself.

Proof. For each $p \in X,\left(g_{i}(p)\right)_{i>0}$ is a Cauchy sequence since $g_{i}(p)$ and $h_{i+1} \circ g_{i}(p)=g_{i+1}(p)$ lie in some one element of $G_{i}$ and mesh $G_{i}$ $<1 / 2^{i}$. This implies that the limit $\left(g_{i}\right)_{i>0}$ is uniquely defined. Since each $g$ is continuous and $d\left(g_{i}(p), g_{i+1}(p)\right)<1 / 2^{i}$ for all $p$, the limit $\left(g_{i}\right)_{i>0}$ is continuous.

Also, for any $q \in X, q$ and $h_{i+1}^{-1}(q)$ lie in some one element of $G_{i}$. Since mesh $g_{i}^{-1}\left(G_{i}\right)<1 / 2^{i}$, the distance between $g_{i}^{-1}(q)$ and $g_{i}^{-1}$ $\circ h_{i+1}^{-1}(q)=g_{i+1}^{-1}(q)$ is less than $1 / 2^{i}$. Hence $\left(g_{i}^{-1}(q)\right)_{i>0}$ converges. Indeed $\left(g_{i}^{-1}\right)_{i>0}$ converges to a continuous function and thus $\left(g_{i}\right)_{i>0}$ converges to a homeomorphism of $X$ onto itself.

The following is a variation of Theorem 4.2 that we shall use. If $A, B$ are sets we use $d(A, B)$ to denote the greatest lower bound of $d(a, b)$ where $a \in A, b \in B$.

Theorem 4.3. Suppose $\left(K_{i}\right)_{i>0}$ is a sequence of closed sets in a complete metric space $X$ and for each positive integer $i, h_{i}$ is a homeomorphism of $X \backslash h_{i-1} \circ \cdots \circ h_{2} \circ h_{1} \circ \operatorname{Id}\left(K_{i} \backslash \bigcup_{1}^{i-1} K_{j}\right)$ onto $X$. Let $G_{i}$ be an open covering of $X \backslash \bigcup_{1}^{i-1} K_{j}$ such that mesh $G_{i}<1 / 2^{i}$, mesh $h_{i}$ $\circ \cdots \circ h_{2} \circ h_{1}\left(G_{i}\right)<1 / 2^{i}$, and if $g \in G_{i}$, diameter $g<d\left(g, \bigcup_{1}^{i-1} K_{j}\right) / 2^{i}$. Then $\left(h_{i} \circ \cdots \circ h_{2} \circ h_{1}\right)_{i>0}$ converges to a homeomorphism of $X \backslash \bigcup K_{i}$ onto $X$ if each $h_{i+1}$ is limited by $h_{i} \circ \cdots \circ h_{2} \circ h_{1}\left(G_{i}\right)$.

The proof is similar to the proof of Theorem 4.2. The condition that diameter $g<d\left(g, \cup_{1}^{i-1} K_{j}\right) / 2^{i}$ is used to show that $\left(h_{1}^{-1} \circ h_{2}^{-1} \circ \cdots \circ h_{i}^{-1}(q)\right)_{i>0}$ converges to a point of $X \backslash \bigcup K_{j}$ rather than to a point of $U K_{j}$.

5. Isotopies. Several times in this paper we shall be concerned with isotopies, which we define for our purposes as follows. For a metric space $X$, an isotopy $H$ of $X$ onto $X$ is a continuous 1-parameter family of homeomorphisms $H_{t}(0 \leqq t \leqq 1)$ of $X$ onto $X$ such that $H_{0}=\mathrm{Id}$; it is required that $H$ be simultaneously continuous in $t$ and $X$ rather than that homeomorphisms be near each other.

One can think of an isotopy as a motion of $X$ onto itself starting with the identity $H_{0}$, ending with $H_{1}$, and using $t$ as a time variable. 
Although it is required that an isotopy $H$ be continuous in both $t$ and $X$, it is not ordinarily required that $\left\{H_{t}^{-1}\right\}_{0 \leq t \leq 1}$ be continuous in this sense. If it is, we call the isotopy invertible. In this paper we shall only use isotopies that are invertible.

For compact spaces, all isotopies are invertible, but this is not true for spaces in general. A noninvertible isotopy $H$ of $l_{2}$ onto itself can be defined as follows. Let $f_{i}$ be a map of $[0,1]$ onto $[1 / i, 1]$ such that $f_{i}[0,1 / i+1]=f_{i}[1 / i, 1]=1$. For each $\left(x_{1}, x_{2}, \cdots\right) \in l_{2}$, let $H_{t}\left(x_{1}, x_{2}, \cdots\right)=\left(x_{1} \cdot f_{1}(t), x_{2} \cdot f_{2}(t), \cdots\right)$. Although $H_{0}^{-1}$ is continuous in $l_{2}$ and $H_{t}^{-1}(0,0, \cdots)$ is continuous in $t, H^{-1}$ is not continuous in $t$ and $l_{2}$ at $t=0,(0,0, \cdots) \in l_{2}$.

For $K \subset X$, an invertible isotopy $H$ pushing $K$ off $X$ is a 1-parameter family of homeomorphisms $H_{t}(0 \leqq t \leqq 1)$ onto $X$ such that

$$
\begin{aligned}
& H_{0}=\mathrm{Id}, \\
& H_{t}(X)=X \text { for }(0 \leqq t<1), \\
& H_{1}(X \backslash K)=X,
\end{aligned}
$$

and

$$
H \text { and } H^{-1} \text { are continuous in } t \text { and } X \text {. }
$$

Those who prefer to think of an isotopy as a map $H$ of $X \times[0,1]$ onto $X$ that is an onto homeomorphism at every level may regard this invertible isotopy pushing $K$ off $X$ as a map from $(X \times[0,1]) \backslash(K \times\{1\})$ onto $X$ satisfying the appropriate conditions.

If $K$ is a single point $p$, we say that $H$ is an invertible isotopy pushing $p$ off $X$.

In Lemma 7.1 and Step 1 of Lemma 8.2 we exhibit invertible isotopies pushing points off $s$ and a copy $l_{2}(1)$ of $l_{2}$. The motions in the two cases are rather different. The first pushes a point off $s$ in some one direction while the second pushes a point off $l_{2}(1)$ by increasing its norm (distance from the origin) using infinitely many directions. In both instances, the isotopies pushing individual points off the spaces are the key devices in pushing certain countable unions of closed sets of infinite deficiency off $s$ and $l_{2}(1)$.

For any isotopy $H$ on a space $X$ and numbers $a, b(0 \leqq a<b \leqq 1)$, we let $H[a, b]$ denote the isotopy which acts on the interval $[a, b]$ as $H$ acts on $[0,1]$. Specifically,

$$
H[a, b]_{t}=\left\{\begin{array}{l}
\operatorname{Id} \text { for } 0 \leqq t \leqq a \\
H_{1} \text { for } b \leqq t \leqq 1 \\
H_{(t-a) /(b-a)} \text { for } a \leqq t \leqq b .
\end{array}\right.
$$


Observe that for $H$ and $F$ isotopies on $X$, a motion which is essentially $H$ followed by $F$ may be represented as the isotopy defined for each $t(0 \leqq t \leqq 1)$ and any $a, b, c(0 \leqq a<b<c \leqq 1)$ as $F[b, c]_{t} \circ H[a, b]_{t}$.

The following proposition is a variation of Theorem 4.1.

LEMмA 5.1. Suppose that for each positive integer $i, H^{i}$ is an invertible isotopy of $X$ onto $X$ and $p_{0} \in X$. Then

$$
H=\left\{L \prod_{i>0} H^{i}[1-(1 / i), 1-(1 / i+1)]_{t}\right\}
$$

is an invertible isotopy pushing $p_{0}$ off $X$ provided that

(1) for each $p \in X \backslash\left\{p_{0}\right\}$, there is a neighborhood $U$ of $p$ in $X$ and an integer $n(U)$ such that for each $n>n(U), H_{t}^{n}=\mathrm{Id}$ on $H_{1}^{n(U)} \circ \ldots$ $\circ H_{1}^{2} \circ H_{1}^{1}(U)$ and

(2) for each $q \in X$, there is a neighborhood $V$ of $q$ in $X$ and an integer $n(V)$ such that for each $n>n(V),\left(H_{t}^{n}\right)^{-1}=\mathrm{Id}$ on $V$ and $\left(H_{1}^{n(V)} \circ \cdots \circ H_{1}^{2} \circ H_{1}^{1}\right)^{-1}(V) \subset X \backslash\left\{p_{0}\right\}$.

Proof. For any $t(0 \leqq t<1)$, let $i$ be such that $1-(1 / i)>t$. Then $H^{i}[1-(1 / i), 1-(1 / i+1)]_{t}$ is the identity. Hence $H_{t}$ may be considered as a finite product of homeomorphisms of $X$ onto itself. Hence, the continuity of $H$ and $H^{-1}$ for values of $t$ in $(0 \leqq t<1)$ follows from the continuity of the finite factors to be considered.

For $x_{0} \in X \backslash\left\{p_{0}\right\}$ and $t=1$, the continuity of $H$ at $\left(x_{0}, 1\right)$ follows from Condition 1 in the statement of the lemma while if $x \in X$, the continuity of $H^{-1}$ at $(x, 1)$ follows from Condition 2.

6. Straightening sets in $s$. There are two related ways of showing that if a countable collection of compact sets are pushed out of $s$, the remainder is homeomorphic with $s$. In one of these procedures, $s$ is regarded as the pseudo interior of the Hilbert cube. This approach was used in [2]. The procedure used in [2] offers an alternative (and perhaps easier) method of proving Corollary 9.4. However, in the present paper we adopt a procedure that parallels our treatment of the proof that $l_{2} \sim l_{2} \backslash \cup E^{i}$. In $\$ \S 6$ and 7 we regard $s$ as $\prod_{i>0} R_{i}$ where $R_{i}$ denotes a copy of the real line. In this section we show how to move sets into such nice positions that they can be pushed out of $s$ with a procedure to be described in $\$ 9$.

Lemma 6.1. For any compact subset $C$ of $s$ there exists a homeomorphism $h$ of $s$ onto $s$ such that $h(C)$ projects onto a single point of $R_{1}-$ that is, $\tau_{1} h(C)$ is a single element. 
Proof. The proof is in two steps. In the first step we adjust $C$ so that its image intersects any line parallel to the $R_{1}$-axis in at most one point. In the second step we move points in the 1st direction so that the final image of $C$ lies in a hyperspace of $s$ perpendicular to the $R_{1}$-axis.

Step 1. For each $i>1$, let $f_{i}$ be a homeomorphism of $R_{1} \times R_{i}$ onto itself such that (1) $f_{i}$ does not change the 1 st coordinate of any point and (2) for some closed rectangular region $D_{i}$ in $R_{1} \times R_{i}$ with $\tau_{\{1, i\}}(C) \subset D_{i}, f_{i}\left(D_{i}\right)$ is a closed parallelogram region intersecting no line parallel to the $R_{1}$-axis in a set of diameter greater than $1 / 2^{i}$.

Let $f$ be the homeomorphism of $s$ onto itself such that $f\left(x_{1}, x_{2}, \ldots\right)$ $=\left(x_{1}, y_{2}, y_{3}, \cdots\right)$ where $y_{i}$ is the 2nd coordinate of $f_{i}\left(x_{1}, x_{i}\right)$. Each point of $s$ has a unique image in $s$ and a unique inverse image in $s$ determined coordinatewise. Continuity follows from the continuity of coordinate functions determined by the $f_{i}$ 's.

For any $i>0$, we know from the definition of $f_{i}$ that $f(C)$ cannot intersect any line parallel to the $R_{1}$-axis in a set of diameter more than $1 / 2^{i}$. Therefore $f(C)$ intersects any such line in at most a single point.

Step 2. Let $s(0)$ be the set of points in $s$ whose 1 st coordinate is 0 and $\tau^{*}$ denote the projection function of $s$ onto $s(0)$. Then $\tau^{*} \mid f(C)$ is a homeomorphism into $s(0)$.

Let $\phi: \tau^{*}(f(C)) \rightarrow R_{1}$ be defined by $\phi=\tau_{1} \tau^{*-1} \mid \tau^{*}(f(C))$. By the Tietze Extension Theorem there is a map $\Phi: s(0) \rightarrow R_{1}$ such that $\Phi \mid \tau^{*}(f(C))=\phi$.

Let $h^{*}$ be the homeomorphism of $s$ onto itself such that for each point $p \in s(0)$ and line $L_{p}$ through $p$ parallel to the $R_{1}$-axis, $h^{*} \mid L_{p}$ is a translation of $-\Phi(p)$ units in the 1st direction. The homeomorphism promised by Lemma 6.1 is $h=h^{*} \circ f$. Note that $h(C) \subset s(0)$ and thus $\tau_{1}(h(C))=0$.

Theorem 6.2. For each collection $\left\{C_{i}\right\}_{i>0}$ of compact subsets of $s$ there is a homeomorphism $g$ of $s$ onto s such that each $g\left(C_{i}\right)$ is infinitely deficient.

Proof. Let $\alpha_{1}, \alpha_{2}, \ldots$ be disjoint infinite subsets of $Z$ such that $\bigcup \alpha_{i}=Z$. Let $s$ be written as $\prod_{i>0} s^{\alpha_{i}}$ where $s^{\alpha_{i}}=\prod_{j \in \alpha_{i}} R_{j}$.

Let $\theta: Z \rightarrow Z$ be such that for each $i>0, \theta^{-1}(i)$ is infinite. We regard each $s^{\alpha_{i}}$ as a copy of $s$ and learn from Lemma 6.1 that there is a homeomorphism $g_{i}$ of $s^{\alpha_{i}}$ onto itself such that $g_{i}\left(\tau_{\alpha_{i}}\left(C_{\theta(i)}\right)\right)$ is deficient with respect to the first element of $\alpha_{i}$.

Let $g$ be the homeomorphism of $s=\prod_{i>0} s^{\alpha_{i}}$ onto itself defined coordinatewise as $g_{i}$ on $s^{\alpha_{i}}$. From the definitions of $\theta$ and of the $g_{i}$ 's it follows that for each $i>0, g\left(C_{i}\right)$ is infinitely deficient. 
7. Isotopies pushing points off $s$. We shall need to consider a 2-parameter $(0<r \leqq 1,0 \leqq t \leqq 1)$ family of homeomorphisms ${ }_{r} H_{t}$ such that for $r$ fixed, ${ }_{r} H$ is an isotopy pushing the origin off $s$. We call such a family an invertibly continuous family of invertible isotopies if both ${ }_{r} H_{t}$ and ${ }_{r} H_{t}^{-1}$ are continuous in $r, t$, and $s$. We shall want each ${ }_{r} H_{t}$ to be the identity outside the $r$-neighborhood of the origin in $s$. We are supposing that $s$ has the complete metric defined by $d(x, y)$ $=\sum\left(\min \left(1 / 2^{i},\left|x_{i}-y_{i}\right|\right)\right)$.

We first describe an invertible isotopy pushing the origin off $s$ and then modify the isotopy to obtain invertibly continuous families of invertible isotopies each pushing the origin off $s$.

Lemma 7.1. There is an invertible isotopy $H$ pushing the origin $p_{0}$ off $s$.

Proof. We shall push $p_{0}$ off $s$ along the positive ray of the $x_{1}$-axis. For convenience, we regard each finite product of lines as having a Euclidean metric.

For each positive integer $i$, let $F^{i}$ be an invertible isotopy on $R_{1} \times R_{i+1}$ such that (1) $F_{i}^{i}$ leaves each point whose 1 st coordinate is less than $i-1-1 / 2^{i-1}$ fixed while (2) $F_{1}^{i}$ carries the vertical ray from $\left(i-1,-1 / 2^{i}\right)$ through $(i-1,1)$ isometrically onto the horizontal ray from $\left(i-1 / 2^{i}, 0\right)$ through $(i+1,0)$. The isotopy $F^{i}$ can result from a "limited rotation" about $(i-1,0)$ (not all points of the plane being rotated) followed by a "limited translation."

Let $a \in R_{2} \times \cdots \times R_{i}, b \in R_{1} \times R_{i+1}$, and $c \in \prod_{j>i+1} R_{j}$. For any $i>1$ and any $a \in R_{2} \times \cdots \times R_{i}$ let $\phi_{i}(a)=\max \left\{0,1-2^{i+1} \cdot d(0, a)\right\}$ where $d(0, a)$ denotes the distance from $a$ to the origin of Euclidean (i-1)-space $R_{2} \times \cdots \times R_{i}$. To avoid a special argument, we suppose that $\phi_{1}$ is the constant 1 . Let $H^{i}$ be an isotopy on $s$ defined for each $t(0 \leqq t \leqq 1)$ as

$$
H_{t}^{i}(a, b, c)=\left(a, F_{t \cdot \phi_{i}(a)}^{i}(b), c\right) .
$$

When we verify that $H^{1}, H^{2}, \cdots$ satisfies Conditions 1 and 2 of the statement of Lemma 5.1, it will follow from that lemma that

$$
H=\left\{L \prod_{i>0} H^{i}[1-1 / i, 1-1 / i+1]_{t}\right\}
$$

is an invertible isotopy pushing $p_{0}$ off $s$. Let $h^{i}$ denote $H_{1}^{i} \circ \cdots$ $\circ H_{1}^{2} \circ H_{1}^{1}$.

(1) To see that the $H^{i}$ 's satisfy Condition 1 , one need only consider the geometry of the successive motions. Specifically, we let $p \in s \backslash p_{0}$ with $p=\left(0, \cdots, a_{j}, a_{j+1}, \cdots\right)$ where $a_{j}$ is the first nonzero coordinate of $p$. Let $h^{j}(p)=\left(b_{1}, b_{2}, \cdots, b_{j}, b_{j+1}, a_{j+2}, \cdots\right)$ and $h^{j+1}(p)$ 
$=\left(c_{1}, b_{2}, \cdots, b_{j+1}, c_{j+2}, a_{j+3}, \cdots\right)$. If $j>1$, each of $b_{2}, \cdots, b_{j}$ is zero but we shall not use this information. If any one of $b_{2}, \cdots$, $b_{j+1}, c_{j+2}$ is nonzero, there is a neighborhood $U$ of $p$ and an integer $k$ such that $\phi_{k}=0$ on the projection of $h^{k}(U)$ into $R_{2} \times \cdots \times R_{k}$. Hence for $k^{\prime} \geqq k, H_{\imath}^{k^{\prime}}=$ Id on $h^{k}(U)$. If each of $b_{2}, \cdots, b_{j+1}$ is zero, then $b_{1} \neq j$ since $h^{j}\left(0, \cdots, 0, a_{j+2}, \cdots\right)=\left(j, 0, \cdots, 0, a_{j+2}, \cdots\right)$. If $b_{1} \neq j$ and $c_{j+2}=0, c_{1}<j+1-1 / 2^{j+1}$ since $F_{1}^{j+1}$ takes the ray from $\left(j,-1 / 2^{j+1}\right)$ through $(j, 1)$ onto the ray from $\left(j+1-1 / 2^{j+1}, 0\right)$ through $(j+2,0)$. But if $c_{1}<j+1-1 / 2^{j+1}$, there is a neighborhood $U$ of $p$ such that the first coordinate of each point of $h^{j+1}(U)$ is less than $j+1-1 / 2^{j+1}$. Then for $k \geqq j+1, H_{t}^{k}=\operatorname{Id}$ on $h^{j+1}(U)$.

(2) To see that the $H^{i}$ 's satisfy Condition 2 , it is only necessary to notice that $H_{t}^{i+1}$ does not move a point unless its first coordinate is greater than $i-1 / 2^{i}$ and the first coordinate of $h^{i}\left(p_{0}\right)$ is $i$.

The following lemma is included as a stepping stone toward Lemma 7.3.

Lemma 7.2. Suppose $s$ is expressed as $s=s^{\alpha} \times s^{\alpha^{\prime}}$ as suggested in Lemma 2.1 where $s^{\alpha}, s^{\alpha^{\prime}}$ are copies of $s$ with origins $p_{0}^{\alpha}, p_{0}^{\alpha^{\prime}}$. Then if $H$ is an invertible isotopy pushing $p_{0}^{\alpha}$ off $s^{\alpha}$ and $\phi_{r}$ is a continuous 1-parameter $(0<r \leqq 1)$ family of maps of $s^{\alpha^{\prime}}$ into $[0,1]$ such that for each $r$, $\phi_{r}^{-1}(1)=p_{0}^{\alpha^{\prime}}$, then

$$
{ }_{r} H_{t}(p, q)=\left(H_{t \cdot \phi_{r}(\alpha)}(p), q\right)
$$

defines an invertibly continuous 1 -parameter $(0<r \leqq 1)$ family of invertible isotopies each pushing the origin off $s$.

The proof is left to the reader.

LEMMA 7.3. There is an invertibly continuous 1 -parameter $(0<r \leqq 1)$ family of invertible isotopies ${ }_{r} H$ each pushing the origin $p_{0}$ off s such that ${ }_{r} H_{t}$ is the identity outside the $r$-neighborhood of $p_{0}$.

Proof. For any $r(0<r \leqq 1),{ }_{r} H$ is to be an isotopy whose "action" pushes the origin off a certain subspace $s_{0}$ of $s$ and is "phased out" in a neighborhood $U$ of the origin in the complementary subspace $s_{0}^{\prime}$ (i.e., $\left.s=s_{0} \times s_{0}^{\prime}\right)$. The two "factors" which determine the size of the domain of support of the isotopy ${ }_{r} H$ are (1) the diameter of the subspace $s_{0}$ and (2) the diameter of the neighborhood $U$ of the origin in $s_{0}^{\prime}$. We shrink the former, as $r$ tends to zero, by a process of swapping axes and the latter by a reduction process introduced after each swap of axes.

Let $\alpha$ be an infinite subset of $Z$ such that $\alpha^{\prime}$ is infinite and $1,2,3 \in \alpha^{\prime}$. The distances in $s^{\alpha}$ and $s^{\alpha^{\prime}}$ are given by $d(x, y)$ 
$=\sum_{i \in \alpha}\left(\min \left(1 / 2^{i},\left|x_{i}-y_{i}\right|\right)\right)$ and $d(x, y)=\sum_{j \in \alpha^{\prime}}\left(\min \left(1 / 2^{i},\left|x_{j}-y_{j}\right|\right)\right)$ respectively. Note that the diameter of $s^{\alpha}$ is less than $1 / 2^{3}$.

Let $H$ be an isotopy pushing the origin off $s^{\alpha}$ as guaranteed by Lemma 7.1.

Let $\phi$ be a map of $s^{\alpha^{\prime}}$ onto $[0,1]$ such that $\phi^{-1}(1)=p_{0}^{\alpha^{\prime}}$ and $\phi=0$ for all points outside a 1/8-neighborhood of $p_{0}^{\alpha^{\prime}}$. We could have specified that $\phi(q)=\max \left[0,1-8 \sum_{i \in \alpha^{\prime}}\left(\min 1 / 2^{i},\left|x_{i}\right|\right)\right]$ for $q=\left(x_{n_{1}}, x_{n_{2}}, \ldots\right)$ but we shall not be concerned with the particular description of $\phi$. In fact, it is perhaps best not to define $\phi$ in terms of the distance from $p_{0}^{\alpha^{\prime}}$ since this distance changes when coordinates are interchanged. We define ${ }_{1} H$ so that for each $(p, q) \in s^{\alpha} \times s^{\alpha^{\prime}}$ (with $(p, q) \neq p_{0}$ for $t=1$ )

$$
{ }_{1} H_{t}(p, q)=\left(H_{t \cdot \phi(q)}(p), q\right) .
$$

Note that ${ }_{1} H_{t}(p, q)=(p, q)$ if $d\left((p, q), p_{0}\right) \geqq 1 / 4$. This is because $1,2,3 \in \alpha^{\prime}$ and $d\left((p, q), p_{0}\right) \geqq 1 / 4$ implies $d\left(q, p_{0}^{\alpha^{\prime}}\right) \geqq 1 / 8$ and $\phi(q)=0$.

As $r$ changes from 1 to $1 / 2$, we define ${ }_{r} H$ by modifying ${ }_{1} H$ by swapping axes and reducing $\phi$. We describe each of these operations separately.

Swapping axes. Our purpose of swapping axes is to move the small positive integers out of $\alpha$ and hence reduce the diameter of $s^{\alpha}$. We recall that 1,2, 3 are in $\alpha^{\prime}$. Let $j$ be the least integer in $\alpha$ and $k$ be an integer in $\alpha^{\prime}$ larger than $j$. Let $\alpha_{1}^{\prime}$ be the subset of $Z$ obtained from $\alpha^{\prime}$ by replacing $k$ by $j$ and $\alpha_{1}$ be the subset obtained from $\alpha$ by replacing $j$ by $k$.

The action of the isotopy ${ }_{1} H$ is defined on $s^{\alpha}$ and is phased out in a neighborhood of the origin in $s^{\alpha^{\prime}}$. We gradually transfer the action over $3 / 4 \leqq r \leqq 1$ so that the action of ${ }_{3 / 4} H$ will be defined on $s^{\alpha_{1}}$ and be phased out in a neighborhood of the origin in $s^{\alpha 1^{\prime}}$. The following formulas describe a suitable version of this process.

For $0 \leqq \lambda \leqq 1$, let $F_{\lambda}$ be the rotation of $s$ such that if $F_{\lambda}\left(x_{1}, x_{2}, \cdots\right)$ $=\left(y_{1}, y_{2}, \cdots\right)$, then $y_{i}=x_{i}$ unless $i \in\{j, k\}$ and $\left(y_{j}, y_{k}\right)$ is the image of $\left(x_{j}, x_{k}\right)$ under a clockwise rotation of the plane $R_{j} \times R_{k}$ by $\lambda \cdot \pi / 2$ about the origin. Let $f(r)$ be the linear function that sends 1 to 0 and $3 / 4$ to 1 . Then for $3 / 4 \leqq r \leqq 1$,

$$
{ }_{r} H_{t}=F_{f(r)}^{-1} \circ{ }_{1} H_{t} \circ F_{f(r)} .
$$

We note that if $d\left(x, p_{0}\right) \geqq 1 / 2$, then ${ }_{r} H_{t}(x)=x$ if $3 / 4 \leqq r \leqq 1$. This is because for such $x d\left(F_{f(r)}(x), p_{0}\right) \geqq 1 / 2-1 / 2^{i} \geqq 1 / 4,{ }_{1} H_{\imath} \circ F_{f(r)}(x)$ $=F_{f(r)}(x)$ and $F_{f(r)}^{-1} \circ{ }_{1} H_{t} \circ F_{f(r)}(x)=x$.

Reducing $\phi$. Here we define ${ }_{r} H$ for $1 / 2 \leqq r \leqq 3 / 4$. Let $x=\left(p^{\prime}, q^{\prime}\right)$ 
where $p^{\prime} \in s^{\alpha_{1}}, q^{\prime} \in s^{\alpha_{1}{ }^{\prime}}$. From the definitions of ${ }_{1} H$ and $F_{\lambda}$ and specifically from the fact that the rotation of $R_{j} \times R_{k}$ which determines $F_{1}$ merely interchanged vertical and horizontal lines in $R_{j} \times R_{k}$, it follows that

$$
{ }_{3 / 4} H_{t}\left(p^{\prime}, q^{\prime}\right)=\left(H_{t^{\prime} \cdot \phi_{3 / 4}}\left(q^{\prime}\right)\left(p^{\prime}\right), q^{\prime}\right)
$$

where $\phi(3 / 4)$ is defined below and where $H_{t}^{\prime}$ is $T^{-1} \circ H_{t} \circ T$ applied to $s^{\alpha_{1}}$ instead of $s^{\alpha}$ with $T$ being a permutation of a finite number of coordinates. The reason for introducing $T$ is that $k$ may not be the least integer in $\alpha_{1}$ and in this case we need to shift $k$ to the first element of $\alpha_{1}$ and to move all the others in $\alpha_{1}$ which are less than $k$ into their successors in $\alpha_{1}$. Also, $\phi_{3 / 4}$ is a map of $s^{\alpha_{1}^{\prime}}$ onto $[0,1]$ such that $\phi_{3 / 4}^{-1}(1)=p_{01}^{\alpha^{\prime}}$ and $\phi_{3 / 4}=0$ except in a small neighborhood of the origin $p_{0^{1}}^{\alpha}$. In fact, $\phi_{3 / 4}=\phi \circ T^{\prime}$ where $T^{\prime}$ is the map from $s^{\alpha_{1}^{\prime}}$ on to $s^{\alpha^{\prime}}$ which merely moves the $j$ th coordinate of a point to the $k$ th coordinate and changes its sign. Note that if $d\left(x, p_{0}\right) \geqq 1 / 2$, then $\phi_{3 / 4}\left(q^{\prime}\right)=0$ as $d\left(q^{\prime}, \quad p_{0}^{\alpha^{\prime}}\right) \geqq 1 / 2-1 / 16$ and $d\left(T^{\prime}\left(q^{\prime}\right), p_{0}^{\alpha^{\prime}}\right) \geqq 1 / 2-1 / 16-1 / 32 \geqq 1 / 8$ which implies that $\phi_{3 / 4}\left(q^{\prime}\right)=\phi\left(T^{\prime}\left(q^{\prime}\right)\right)=0$.

Let $\phi_{1 / 2}$ be a map of $s^{\alpha_{1}^{\prime}}$ onto $[0,1]$ so that $\phi_{1 / 2} \leqq \phi_{3 / 4}, \phi_{1 / 2}^{-1}(1)=p_{0}^{\alpha^{\prime}}$, and $\phi_{1 / 2}=0$ except in the $1 / 2^{4}$-neighborhood of $p_{0^{2}}^{\alpha^{\prime}}$. For each $q^{\prime} \in s^{\alpha_{2}^{\prime}}$ let, $\phi_{r}\left(q^{\prime}\right)$ divide $\phi_{1 / 2}\left(q^{\prime}\right), \phi_{3 / 4}\left(q^{\prime}\right)$ in the same ratio that $r$ divides $1 / 2$, $3 / 4$ with $\phi_{r}\left(q^{\prime}\right)=\phi_{1 / 2}\left(q^{\prime}\right)$ if $\phi_{1 / 2}\left(q^{\prime}\right)=\phi_{3 / 4}\left(q^{\prime}\right)$. Then for $1 / 2 \leqq r \leqq 3 / 4$, define

$$
{ }_{r} H_{t}\left(p^{\prime}, q^{\prime}\right)=\left(H_{t \cdot \phi_{r}\left(q^{\prime}\right)}\left(p^{\prime}\right), q^{\prime}\right) \text {. }
$$

As $r$ moved from 1 to $1 / 2$, we defined ${ }_{r} H_{t}$ from ${ }_{1} H_{t}$ by swapping axes and reducing $\phi$. By similarly swapping axes so as to replace the least element of $\alpha_{1}$ with a larger element of $\alpha_{1}^{\prime}$ and by further reducing $\phi$, we define ${ }_{r} H_{t}(1 / 4 \leqq r \leqq 1 / 2)$ from ${ }_{1 / 2} H_{t}$ in an analogous fashion. Similarly, we define ${ }_{r} H_{t}$ as $r$ shrinks to $1 / 2^{3}$, to $1 / 2^{4}, \cdots$.

8. Isotopies pushing points off $l_{2}$. We prove lemmas about pushing points off $l_{2}$ similar to those we proved about pushing points out of $s$ in the last section. We recall that $S_{1}$ is the set of all points of $l_{2}$ at a distance of 1 from the origin.

Lemma 8.1. There is an invertible isotopy $F$ pushing a point off $S_{\mathbf{1}}$.

Proof. Let $l_{2}(1)$ be the set of all points of $l_{2}$ whose first coordinate is 1 . For each positive integer $i$, let $p_{i}$ denote the point of $l_{2}$ whose first $i$ coordinates are each 1 and whose other coordinates are 0 . We prove Lemma 8.1 in two steps.

Step 1. In this step we exhibit a particular invertible isotopy $H$ 
on $l_{2}(1)$ pushing $p_{1}$ out of $l_{2}(1)$. The plan is to push $p_{1}$ to $p_{2}$, then to $p_{3}$, then to $p_{4}, \cdots$, and hence off $l_{2}$. We take care that no point other than $p_{1}$ undergoes more than a finite number of these pushes.

For each integer $i \geqq 2$, let $U_{i}$ be the neighborhood of $p_{i}$ in $l_{2}(1)$ such that

$$
U_{i}=\left\{\left(1, x_{2}, \cdots\right) \in l_{2}(1) \mid \sum_{2}^{i}\left(1-x_{j}\right)^{2}<1 / i^{2}\right\} .
$$

Let $H^{1}$ be the isotopy on $l_{2}(1)$ such that $H_{t}^{1}$ is a translation of $t$ units in the $x_{2}$ direction. For each integer $i \geqq 2, H^{i}$ is the isotopy on $l_{2}(1)$ such that $H_{t}^{i}(p)$ is the point resulting from translating $p$ by $i \cdot t \cdot d\left(p,\left(l_{2}(1) \backslash U_{i}\right)\right)$ in the $x_{i+1}$ direction. Then

$$
H_{t}=L \prod_{i>0} H^{i}[1-1 / i, 1-1 /(i+1)]_{t} .
$$

It may be shown with the use of Theorem 4.1 and Lemma 5.1 that $H$ is an invertible isotopy pushing $p_{1}$ out of $l_{2}(1)$.

Step 2. In this step we describe an invertible isotopy $F$ pushing $p_{1}$ off $S_{1}$. Let $S_{1}^{+}$be the set of all points of $S_{1}$ with positive 1st coordinate and $\rho$ be the projection of $S_{1}^{+}$on to $l_{2}(1)$ from the origin. The invertible isotopy $F$ promised by Lemma 8.1 may be defined as

$$
\begin{aligned}
F_{t}(p) & =\rho^{-1} \circ H_{t} \circ \rho(p) \quad \text { if } p \in S_{1}^{+}, \\
& =p \quad \text { if } p \in S_{1} \backslash S_{1}^{+} .
\end{aligned}
$$

To complete the proof we need only verify the continuity of $F$ and $F^{-1}$ at points $p \in S_{1}$ whose 1 st coordinate is 0 . Suppose the $i$ th coordinate of $p$ is not 0 and $\delta$ is one third the absolute value of this coordinate. A calculation shows that if $U$ is the $\delta$-neighborhood of $p$ in $S_{1}$ and $q \in \rho\left(U \cap S_{1}^{+}\right)$, then the absolute value of the $i$ th coordinate of $q$ is greater than 2 . For $k \geqq i, H_{t}^{k}$ and $\left(H_{t}^{k}\right)^{-1}$ do not move $q$. Hence, to verify the continuity of $F$ and $F^{-1}$, we only need to check the effect of a finite number of $\rho^{-1} \circ H_{t}^{t} \circ \rho^{\prime}$ s near $p$; in fact we only need to check one of them since the composition of a finite collection of continuous functions is continuous.

A computation shows that if $p=\left(0, x_{2}, x_{3}, \cdots\right) \in S_{1}$ and $q=\left(y_{1}, y_{2}, \cdots\right) \in S_{1}^{+}$within $\delta$ of $p$, then for some $0 \leqq \theta \leqq 1$,

$$
\rho^{-1} \circ\left(H_{t}^{j}\right)^{-1} \circ \rho(q)=\left(k \cdot y_{1}, k \cdot y_{2}, \cdots, k\left(y_{j}+\theta \cdot y_{1}\right), \cdots\right)
$$

where $k=\left(1+2 \cdot y_{j} \cdot y_{1} \cdot \theta+y_{1}^{2} \cdot \theta^{2}\right)^{-1 / 2}$. If $\delta$ is small, then $y_{1}$ is small and $k$ is near 1 . Hence coordinatewise, $\rho^{-1} \circ\left(H_{\imath}^{\jmath}\right)^{-1} \circ \rho(q)$ is near $p$. Coordi- 
natewise continuity in $S_{1}$ implies continuity there so $F$ is continuous at $p$. Similarly, $F^{-1}$ is continuous at $p$ also.

LEMma 8.2. There is an invertible isotopy $H$ pushing the origin $p_{0}$ off $l_{2}$ such that each $H_{t}$ is the identity outside the 1-neighborhood of $p_{0}$.

Proof. For each point $p \neq p_{0}$, we let $a_{p}$ denote the projection of $p$ from $p_{0}$ on $S_{1}$.

Let $\phi$ be the map of the nonnegative reals onto $[0,1]$ such that $\phi(x)=0$ if $x \geqq 1, \phi(0)=0, \phi(1 / 2)=1$, and $\phi$ is linear on each of $[0,1 / 2]$ and $[1 / 2,1]$.

The isotopy $H$ promised by Lemma 8.2 is defined by $H_{t}=H^{2}[1 / 2,1]_{t}$ $\circ H^{1}[0,1 / 2]_{t}$ where $H^{1}$ and $H^{2}$ are isotopies defined as follows. The isotopy $H^{1}$ is the identity outside the 1 -neighborhood of $p_{0}$ and $H_{1}^{1}\left(p_{0}\right)=(1 / 2,0,0, \cdots)$. For each $t(0 \leqq t \leqq 1), H_{l}^{2}(p)$ is at the same distance from $p_{0}$ as $p$ and the projection of $H_{t}^{2}(p)$ from $p_{0}$ on $S_{1}$ is $F_{t \cdot \phi\left(d\left(p, p_{0}\right)\right)}\left(a_{p}\right)$ where $F$ is the isotopy of Lemma 8.1.

Lemma 8.3. There is an invertibly continuous 1 -parameter $(0<r \leqq 1)$ family of invertible isotopies ${ }_{r} H$ each pushing the origin $p_{0}$ off $l_{2}$ such that for any $t(0 \leqq t \leqq 1),{ }_{r} H_{t}$ is the identity outside the $r$-neighborhood of $p_{0}$.

Proof. The required isotopy is defined by

$$
{ }_{r} H_{t}=r \circ H_{t} \circ 1 / r
$$

where $H$ is the isotopy of Lemma 8.2 and $r(p)$ is the point whose coordinates are obtained by multiplying those of $p$ by $r$.

9. Eliminating sets with small pushes. Can a small push send a mathematician out of society without leaving a vacancy? We shall be interested in conditions under which a set can be pushed out of a space with a small push.

Lemma 9.1. Let $X$ denote $l_{2}$ or $s, G$ be an open covering of $X, \alpha$ be an infinite subset of $Z$, and $K$ be a closed subset of $X$ which is deficient with respect to $\alpha$. Then for each open set $U$ containing $K$ there exists a homeomorphism $h$ of $X \backslash K$ onto $X$ such that $h$ is limited by $G, h$ is the identity except on $U$, and $\tau_{i}(x)=\tau_{i}(h(x))$ for each $i \in \alpha^{\prime}$.

Proof. We suppose $\alpha^{\prime} \neq \varnothing$ and write $X=X^{\alpha} \times X^{\alpha^{\prime}}$ as suggested by Lemmas 2.1 and 2.2. With no loss of generality we suppose that $\tau_{\alpha}(K)=\left\{p_{0}^{\alpha}\right\}$ where $p_{0}^{\alpha}$ is the origin in $X^{\alpha}$. Let $\tau_{\alpha^{\prime}}(K)=K^{\prime} \subset X^{\alpha^{\prime}}$. See Figure 1.

We need a function $r$ from $X^{\alpha^{\prime}}$ into $(0,1]$ to measure how far each 


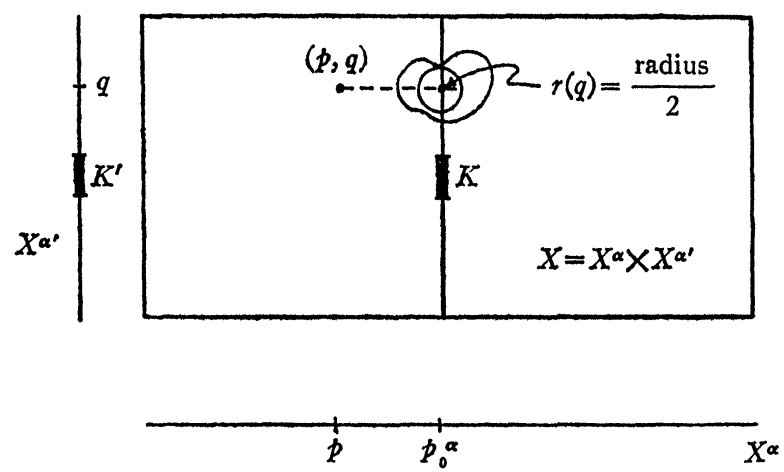

FIGURE 1

point $\left(p_{0}^{\alpha}, q\right)$ lies inside elements of $G$. Let $G^{\prime}$ be an open covering of $X$ of mesh less than 1 such that each element of $G^{\prime}$ lies in an element of $G$ and each element of $G^{\prime}$ intersecting $K$ lies in $U$. Letting $V_{\epsilon}(x)$ denote the $\epsilon$-neighborhood of $x$ in $X$, we define

$$
r(q)=\sup \left\{\epsilon \mid V_{2 \epsilon}\left(p_{0}^{\alpha}, q\right) \text { lies in some element of } G^{\prime}\right\} .
$$

Note that for each $q \in X^{\alpha^{\prime}}, r(q)>0$.

Next, we need a function $t$ from $X^{\alpha^{\prime}}$ into $[0,1]$ to measure the degree of closeness of points of $X^{\alpha^{\prime}}$ to $K^{\prime}$. Let

$$
A=\left\{x \in X^{\alpha^{\prime}} \mid V_{r(x)}\left(p_{0}^{\alpha}, x\right) \nsubseteq U\right\}
$$

and

$$
t(q)=d(q, A) /\left(d(q, A)+d\left(q, K^{\prime}\right)\right) .
$$

(We supposed $A \neq \varnothing$. If $A \neq \varnothing$, define $t(q)=1$.)

Note that $\bar{A} \cap K^{\prime}=\varnothing, t\left(K^{\prime}\right)=1$, and $t(A)=0$.

By Lemma 8.3 for $l_{2}$ (and thus for $l_{2}^{\alpha}$ ) and by Lemma 7.3 for $s$ (and thus for $s^{\alpha}$ ), there exists an invertibly continuous family ${ }_{r} H(0<r \leqq 1)$ of invertible isotopies each pushing $p_{0}^{\alpha}$ off $X^{\alpha}$ and such that for each $t(0 \leqq t \leqq 1),{ }_{r} H_{t}$ is the identity outside the $r$-neighborhood of $p_{0}^{\alpha}$ in $X^{\alpha}$.

We shall verify that the desired homeomorphism $h$ of our lemma is

$$
\left.h(p, q)={ }_{r(q)} H_{t(q)}(p), q\right) .
$$

Since $t(q)=1$ for $q \in K^{\prime}$, we note that for such $q, r(q) H_{t(q)}$ is a homeomorphism of $\left(X^{\alpha} \backslash\left\{p_{0}\right\}\right) \times\{q\}$ onto $X^{\alpha} \times\{q\}$. Also, since $t(q)<1$ for $q \in X^{\alpha^{\prime}} \backslash K^{\prime}$, we note that for such $q,{ }_{r(q)} H_{t(q)}$ is a homeomorphism of 
$X^{\alpha} \times\{q\}$ onto $X^{\alpha} \times\{q\}$. Since $r$ and $t$ are continuous functions on $X$ and ${ }_{r} H$ is a continuous family of isotopies, then $h$ is continuous. Since ${ }_{r} H$ is an invertibly continuous family of invertible isotopies and $h^{-1}$ is described by $\left.h^{-1}(p)={ }_{r(q)} H_{t(q)}^{-1}(p), q\right), h^{-1}$ is also continuous. Hence, $h$ is a homeomorphism of $X \backslash K$ onto $X$.

Since $q$ is the second coordinate of $h(p, q), \tau_{i}(x)=\tau_{i}(h(x))$ for each $i \in \alpha^{\prime}$. We note that $h$ is the identity outside $U$ since ${ }_{r} H_{t}$ is the identity outside the $r$ neighborhood of $p_{0}^{\alpha}$ and $V_{r(q)}\left(p_{0}^{\alpha}, q\right) \subset U$ if $t(q) \neq 0$.

It only remains to observe that $h$ is limited by $G$. Since ${ }_{r} H_{t}$ is the identity except on the $r$-neighborhood of $p_{0}^{\alpha}$ in $X^{\alpha}$, either $h(p, q)$ $=(p, q)$ or each of $p$ and ${ }_{r(q)} H_{t(q)}(p)$ lie within $r(q)$ of $p_{0}^{\alpha}$. In this latter case, the definition of $r(q)$ insures that both $(p, q)$ and $h(p, q)$ lie in an element of $G$ containing $\left(p_{0}, q\right)$.

We are now in a position to prove a theorem having two important corollaries.

THEOREM 9.2. Let $X$ denote either $l_{2}$ or $s, U$ be an open set in $X$, and $\left\{K_{i}\right\}_{i>0}$ be a countable collection of closed sets of $X$ which lie in $U$, with each $K_{i}$ having infinite deficiency. Then there exists a homeomorphism $h$ of $X \backslash \cup K_{i}$ onto $X$ such that $h \mid X \backslash U$ is the identity.

PROOF. By a standard sequential process augmenting one finite set at a time, it is routine to show that there exists a collection $\left\{\alpha_{i}\right\}_{i>0}$ of disjoint infinite subsets of $Z$ such that $K_{i}$ is deficient with respect to $\alpha_{i}$. Using Lemma 9.1 to assert the existence of $h_{i}$, we can inductively define a sequence of homeomorphisms $\left(h_{i}\right)_{i>0}$ and a sequence of coverings $\left(G_{i}\right)_{i>0}$ satisfying the hypotheses of Theorem 4.3 (specifying that $h_{i}$ does not change the $j$-coordinate of any point unless $\left.j \in \alpha_{i}\right)$ so that $L \prod_{i>0} h_{i}$ is a homeomorphism of $X \backslash \cup K_{i}$ onto $X$.

\section{COROLlaRY 9.3. $l_{2} \backslash U E^{i} \sim l_{2}$.}

CoROLlaRy 9.4. If $\left\{C_{i}\right\}_{i>0}$ is a countable family of compact subsets of $s$, then $s \backslash \cup C_{i}$ is homeomorphic to $s$.

Proof. It follows from Theorem 6.2 that there is a homeomorphism $h$ of $s$ onto itself such that each $h\left(C_{i}\right)$ is infinitely deficient. Then Corollary 9.4 follows from Theorem 9.2.

The following corollary may be useful in studying spaces which are locally like $l_{2}$ or $s$.

Corollary 9.5. If $U$ is an open subset of $l_{2}$ and $K_{1}, K_{2}, \cdots$ are compact subsets in $U$, then there is a homeomorphism $h$ of $l_{2} \backslash \cup K_{i}$ onto $l_{2}$ such that $h \mid l_{2} \backslash U$ is the identity. 
Proof. For convenience, we regard $l_{2}$ as $s$. It follows from Theorem 6.2 that there is a homeomorphism $g$ of $s$ onto itself such that each $g\left(K_{i}\right)$ is infinitely deficient. We learn from Theorem 9.2 that there is a homeomorphism $h^{\prime}$ of $s \backslash \cup g\left(K_{i}\right)$ onto $s$ such that $h^{\prime} \mid s \backslash g(U)$ is the identity. Then $h=g^{-1} \circ h \circ g$.

10. Summary of the procedure. In the foregoing we have established the existence of a homeomorphism between $l_{2}$ and $s$ (Theorem 3.1). Our method of attack was to use several intermediate spaces and homeomorphisms as follows:

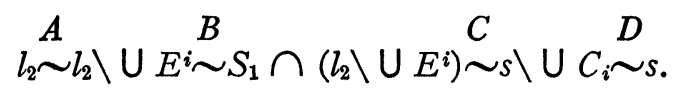

Of the four homeomorphisms, $B$ was easily established in Lemma 3.3 and $C$ was established in Lemma 3.2 by exhibiting explicit formulas. The bulk of the paper was concerned with apparatus for establishing $A$ and $D$ which were stated as Corollaries 9.3 and 9.4. $\$ 4$ identified criteria for the convergence of sequences of homeomorphisms and $\$ 5$ introduced isotopy procedures to be used later.

$\S \S 6$ and 7 were concerned with properties of $s$ while $\$ 8$ was concerned with properties of $l_{2}$ analogous to those of $\$ 7$ for $s$. In this treatment, it appears as if $l_{2}$ is a simpler space to handle than is $s$, but in fact the procedure was designed for $l_{2}$ and then adapted to $s$ since we were unable to adapt to $l_{2}$ a more natural simpler procedure for $s$.

Finally, in $\$ 9$, the results of $\$ \$ 7$ and 8 pushing individual points off $s$ and $l_{2}$ were used to push $U C_{i}$ off $s$ and $U E^{i}$ off $l_{2}$.

11. Some open questions related to $s$ or $l_{2}$. Various topological properties of $s$ or $l_{2}$ have been studied by Klee [13], [14], [15], Anderson [1], [2], [3], [4] and others. In various survey papers to appear in the Proceedings of the Symposium on Infinite Dimensional Topology (Annals of Mathematics Study), such properties are discussed. Here we list a number of open questions suggested conversationally or in print by one or more of several authors including Bessaga, Pelczynski, Klee, Fort, Borsuk, Eells, Palais, Henderson, Corson and others.

In this section we restrict ourselves to questions directly related to $s$ or $l_{2}$ (and thus to separable metric spaces). See Bessaga [6] for many questions concerning homeomorphisms between various topological linear spaces.

Questions Concerning the Hilbert Cube as a Compactification of $s$. Let 
the Hilbert cube, $I^{\infty}$, be written as $\prod_{i>0} I_{i}$ where for each $i>0, I_{i}$ is the closed interval $[-1,1]$. As in $\S 3$, we may regard $s=\prod_{i>0} I_{i}^{o}$ where for each $i>0, I_{i}^{o}$ is the open interval $(-1,1)$. Then $s \subset I^{\infty}$ and $I^{\infty}$ is a compactification of $s$. Indeed, both $s$ and $I^{\infty} \backslash s$ are dense in $I^{\infty}$. A $\beta^{*}$-homeomorphism is a homeomorphism $h$ of $I^{\infty}$ onto itself such that $h(s)=s$ and a $\beta$-homeomorphism is a homeomorphism $h$ of $I^{\infty}$ onto itself such that $h(s) \supset s$. A closed set $K$ in a space $X$ is said to have Property $Z$ if for every nonnull homotopically trivial open set $U$ in $X, U \backslash K$ is nonnull and homotopically trivial.

(1) Let $K \subset I^{\infty}$. What are necessary and sufficient conditions on $K$ (or on $I^{\infty} \backslash K$ ) in order that there exist a homeomorphism of $I^{\infty}$ onto itself carrying $K$ onto $B\left(I^{\infty}\right)$ ? Anderson has recently shown that if $K \supset B\left(I^{\infty}\right)$ then in order that such a homeomorphism exist it is necessary and sufficient that $K$ be the countable union of closed sets with Property $Z$.

(2) A special case of (1) is the following. Let $s \subset M \subset I^{\infty}$. What are necessary and sufficient conditions on $M$ in order for there to exist a $\beta$-homeomorphism $h$ such that $h(s)=M$ ? In particular, is it necessary and sufficient that (a) $I^{\infty} \backslash M$ be the countable union of compact sets and (b) each compact subset of $M$ have Property $Z$ in $M$ ? (Conditions (a) and (b) are clearly necessary.)

(3) A different version of question (2) would ask simply for necessary and sufficient conditions that $s$ and $M$ be homeomorphic.

(4) Let $s_{f}$ and $I_{f}^{\infty}$ be the sets of all points of $s$ and $I^{\infty}$ with only finitely many nonzero coordinates. Characterize the subsets $K$ of $s\left(\right.$ or $\left.I^{\infty}\right)$ for which there exist homeomorphisms of $s\left(\right.$ or $\left.I^{\infty}\right)$ onto itself carrying $K$ onto $s_{f}$ (or $I_{f}^{\infty}$ ).

(5) Regarding $s \subset I^{\infty}$ it is easy to show that there exist homeomorphisms of $s$ onto itself that cannot be extended to $\beta^{*}$-homeomorphisms, but the following question seems more interesting. For any homeomorphism $h$ of $s$ onto itself does there exist a homeomorphism $f$ of $s$ onto itself such that $f^{-1} h f$ can be extended to a $\beta^{*}$-homeomorphism?

Questions on Infinite Products. (6) Is every product of a compact absolute retract by $I^{\infty}$ homeomorphic to $I^{\infty}$ ?

(7) Is every countable infinite product of compact absolute retracts homeomorphic to $I^{\infty}$ ?

A set is a topologically complete absolute retract if it admits a complete metric and is a retract of every metric space in which it is embedded as a closed set.

(8) Is every product of a topologically complete absolute retract by $s$ homeomorphic to $s$ ?

(9) Is every countable infinite product of topologically complete 
absolute retracts (with infinitely many noncompact factors) homeomorphic to $s$ ?

It is reasonable to weaken questions (6) to (8) by replacing "absolute retract" by "contractible finite complex" or a similar condition. In an as yet unpublished paper, Anderson has shown that any countable infinite product of dendrons is homeomorphic to $I^{\infty}$. (A dendron is an acyclic locally connected one-dimensional continuum.) He feels it likely that the methods of the proof of this proposition can be modified to show that any countable infinite product of contractible finite complexes is homeomorphic to $I^{\infty}$.

(10) Is every product of $I^{\infty}$ by a finite connected complex homeomorphic to a product of $I^{\infty}$ by some manifold-with-boundary?

(11) Is every product of $s$ by a finite connected complex homeomorphic to a product of $s$ by some manifold-with-boundary?

Questions on Topological Banach Manifolds. A number of authors have studied the differential topology of so-called Banach manifolds. For our purposes, we consider a topological Banach manifold (T.B.M.) to be a connected separable metric space locally like $s$-that is with an open covering of sets each homeomorphic to $s$. (In effect we are specifying that our Banach manifolds are modeled on some separable infinite dimensional Banach space.)

(12) Can every T.B.M. be imbedded in $s$ as an open set in $s$ ?

(13) If a T.B.M. can be imbedded in $s$ as an open subset of $s$, then the T.B.M. has a local compactification as a metric space admitting a cover by open sets homeomorphic to open subsets of $I^{\infty}$. Furthermore the T.B.M. intersects such open sets in $I^{\infty}$ in the manner induced by the open imbedding. As a weaker version of the imbedding problem of (12), we can ask if such a local compactification of each T.B.M. is possible.

(14) Is every contractible T.B.M. homeomorphic to $s$ ? What about the special case where the T.B.M. is homeomorphic to an open subset of $s$ ?

(15) More generally than in (14), are every two T.B.M.'s that are of the same homotopy type, homeomorphic to each other?

Questions on Unions of Two Sets. (16) Let $M=H \cup K$. If $H, K$, and $H \cap K$ are all homeomorphic to $I^{\infty}$, is $M \sim I^{\infty}$ ?

(17) Let $M=H \cup K$. If $H$ and $K$ are both closed or both open and if $H, K$, and $H \cap K$ are all homeomorphic to $s$, is $M \sim s$ ?

(18) Let $M=H \cup K$. If $H, K$, and $M$ are all homeomorphic to $I^{\infty}$, is $H \cap K \sim I^{\infty}$ ?

(19) Let $M=H \cup K$. If $H$ and $K$ are both closed or both open and if $H, K$, and $M$ are all homeomorphic to $s$, is $H \cap K \sim s$ ?

Questions on Spaces of Closed Subsets. (20) Is the space of all closed 
subsets (with the Hausdorff metric) of an $n$-cell (or of $I^{\infty}$ ) homeomorphic to $I^{\infty}$ ? This is not even known for $n=1$.

(21) Is the space of all subcontinua of an $n$-cell $(n>1)$ or of $I^{\infty}$ homeomorphic to $I^{\infty}$ ?

Questions on Spaces of Homeomorphisms. (22) Is $s$ homeomorphic to the space of all homeomorphisms of a disk onto itself which are the identity on the boundary of the disk? It is known that $s$ is homeomorphic to the space of orientation preserving homeomorphisms of an interval onto itself.

(23) For any geometric $n$-sphere or $n$-cube $X$ and any sufficiently small $\epsilon>0$, is $s$ homeomorphic to the space of all homeomorphisms of $X$ onto itself which are within $\epsilon$ of the identity? The answer is almost certainly yes for $n=1$.

(24) Is $s$ homeomorphic to the space of all homeomorphisms of $I^{\infty}$ on to itself?

\section{REFERENCES}

1. R. D. Anderson, Hilbert space is homeomorphic to the countable infinite product of lines, Bull. Amer. Math. Soc. 72 (1966), 515-519.

2. - Topological properties of the Hilbert cube and the infinite product of open intervals, Trans. Amer. Math. Soc. 126 (1967), 200-216.

3. - On topological infinite deficiency, Michigan Math. J. 14 (1967), 365-383.

4. - On a theorem of Klee, Proc. Amer. Math. Soc. 17 (1966), 1401-1404. 1932.

5. S. Banach, Théorie des opérations linéaires, Monografie Mat. PWN, Warsaw,

6. C. Bessaga, On topological classification of complete linear metric spaces, Fund. Math. 56 (1965), 251-288.

7. C. Bessaga and V. L. Klee, Two topological properties of topological linear spaces, Israel J. Math. 2 (1964), 211-220.

8. - Every non-normable Frechet space is homeomorphic with all its closed convex bodies, Math. Ann. 163 (1966), 161-166.

9. C. Bessaga and A. Pelczynski, Some remarks on homeomorphisms of F-spaces, Bull. Acad. Polon. Sci. Sér. Sci. Math. Astronom. Phys. 10 (1962), 265-270.

10. M. Fréchet, Les espaces abstraits, Gauthier-Villars, Paris, 1928.

11. M. I. Kadec, On topological equivalence of separable Banach spaces, Dokl. Akad. Nauk SSSR 167 (1966), 23-25=Soviet Math. Dokl. 7 (1966), 319-322.

12. - Proof of the topological equivalence of all separable infinite-dimensional Banach spaces, Functional Anal. and Appl. 1 (1967), 53-62.

13. V. L. Klee, Convex bodies and periodic homeomorphisms in Hilbert space, Trans. Amer. Math. Soc. 74 (1953), 10-43.

14. - Some topological properties of convex sets, Trans. Amer. Math. Soc. 78 (1955), 30-45.

15. - A note on topological properties of normed linear spaces, Proc. Amer. Math. Soc. 7 (1956), 673-674.

Louisiana State University, Baton Rouge and UNIVERSITY OF WisCONSIN, MADISON 